\title{
Influence of Axial Compressor Model Simplification and Mesh Density on Surge Margin Evaluation
}

\author{
Rafał Muchowski ${ }^{1,2^{*}}$, Sławomir Gubernat ${ }^{1,2}$ \\ 1 Doctoral School of Engineering and Technical Sciences, Rzeszow University of Technology, \\ al. Powstańców Warszawy 12, 35-959 Rzeszów, Poland \\ 2 MTU Aero Engines Polska Sp. z o.o., Tajęcina 108, 36-002 Jasionka, Poland \\ * Corresponding author's e-mail: rafal.muchowski@mtupolska.com
}

\begin{abstract}
The menace of surge occurrence in the compressors is taken very seriously and its avoidance became a fundamental for the design of any modern jet engines. Nowadays, a problem with appropriate evaluation of the compressor surge margin while considering different simplifications of three-dimensional CFD model is still present. For that purpose, this article presents a comparison between the measurement data and several variants of 3D CFD models characterized by a specific mesh density. To calculate all the results on which the comparisons and conclusions are based, an 8-stage axial compressor is taken into account. Flow conditions of the machine are computed for three part load speeds: The low, the mid and the high one respecting the variable guide vanes schedule fitted to the specific load. For each of speed variants a four mesh configurations were generated: coarse, medium, fine and extra-fine. All speed configurations were treated with two different turbulence models - Wilcox k- $\omega$ and Menter's SST k- $\omega$, giving ultimately 15 CFD models, calculated with the TRACE solver using an initialization based on a circumferentially averaged flow solution delivered by the Streamline Curvature Method. During the study an additional assessment of reference grid independence was performed and the mesh convergence has been achieved. A comparison between turbulence models and the measurement proves that SST turbulence model is not well distributed through the speeds in compare to the measurement data and the Wilcox turbulence model. Inconsistency of sensitivity in the mesh coarsening for different rotational speeds was found. Increasing the mesh roughness level has to be executed for each speed separately. Overall compressor map shows that shift of the Pressure Ratio and the Mass Flow decreases with lower rotational speed. Neglecting the system add-ons like labyrinth sealing volumes, bleed-ports and other leakages has a visible influence on deviations from the measurements. Because of intended future use in design and optimization the "Medium" grid with Wilcox k- $\omega$ turbulence model was chosen, being a good representation of the Rig characteristics with reduction of the computing time.
\end{abstract}

Keywords: jet engine, axial compressor, compressor map, surge margin, CFD, grid dependency.

\section{INTRODUCTION}

The phenomenon of stall and surge is under investigation since first turbomachinery engines comes to the operation [1]. Due to the different types and complexity of stall and surge events there are many scientific works on that topic $[2 \div 4]$ which each of them provide another step further. The definition of Surge Margin (SM) and operating below this limit is extremely important because of safety point of view. The flow fluctuations during surge can induce serious damages and provide to In-Flight Shut Down (IFSD). Nowadays most of commercial aircrafts are equipped with Turbo-Fan engines what leads to continuous improvement in methods of designing and optimizing the compressors [5].

One of the approaches in turbomachinery design is wide use of Computational Fluid Dynamics (CFD) to analyze the flow through the turbomachines such as e.g. axial compressors $[6,7]$. Detailed simulations are time expensive therefore 
design and optimization is commonly performed with use of the meanline code [8], at specific Design Point (DP) [9] or for a single blade [10].

The approach providing possibility of the Surge Margin optimization is the one which can allow designer to evaluate the stability performance along whole operational range. For that purpose CFD methods are necessary to predict the three-dimensional flow in stability limit region. In work of [11] Surge Margin optimization of centrifugal compressor procedure has been performed. For mesh resolution, Grid Convergence Index approach has been applied with one specified turbulence model (EARSM). Created reference shows good agreement with the measurements . Comparing geometry complexity of centrifugal and axial compressors it is necessary to include more details to define the reference model of an axial flow type. Another work [12] has been aiming for stability improvement with use of casing grooves in a transonic axial compressor. One rotational speed has been used to determine grid resolution. SST turbulence model and $\mathrm{y}^{+} \leq 2$ is applied. No details about geometry representation included. In a paper [13] multistage axial compressor mesh sensitivity analysis on various effects has been performed. Middle part of the machine at the Design Point has been taken into account for the study. In [14] another multistage axial compressor has been considered. Detailed model with leakages, bleed-ports and fillets is presented. The mesh study has been conducted for 3 rotational speeds and $\mathrm{IGV}+\mathrm{R} 1$ configuration as a chosen part of the machine for different $y+$ value. Those approaches gives good measurement data fit but due to the level of detailed modelling it is not an efficient way to use it for the optimization process. It is obvious that all small details of the model e.g. fillets, leakage modeling, bleed-ports at near stall conditions have a crucial impact on the stability margin. Ideally would be to create a model without any details and with the coarse mesh which will fit the measurements and be time efficient in terms of optimization sampling.

The objective of this paper is to find a simplified setup providing a good estimation of the Surge Margin in a relatively short time. The results of this work will further be used in a project of MultiFidelity optimization process therefore suitable 3D CFD setup is necessary. In contrast to the existing studies grid analysis are performed for a full eightstage machine and for constant $\mathrm{y}^{+}$. The proposed approach is aiming to avoid geometry discretization quality change and only the through flow mesh density is adjusted. Two turbulence models are tested to check SM fitment with measurements. For three rotational speeds steady RANS (Reynolds-averaged Navier-Stokes) simulations has been performed.

The case considered is a state of the art 8 stage axial High Pressure Compressor experimental rig developed by MTU Aero Engines AG.

\section{CFD COMPUTATIONAL APPROACH}

All three-dimensional simulations presented in this paper were conducted with the TRACE (Turbomachinery Research Aerodynamics Computational Environment) CFD code developed by DLR Cologne. TRACE is a software system for the computation of three-dimensional internal flow of multi-stage compressors and turbine components, solving Reynolds-Averaged Navier-Stokes equations.

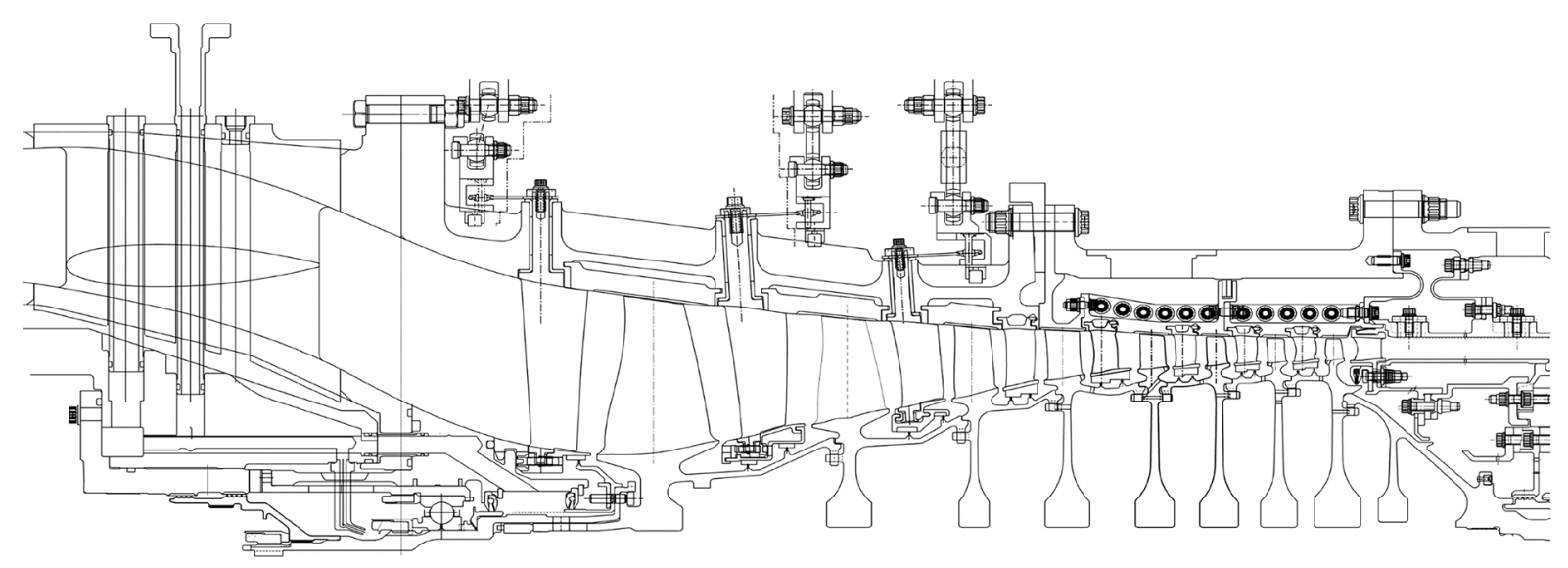

Fig. 1. Cross-sectional view of the compressor 
The 3D model is defined as a multi-stage Rotor-Stator domain between two Measurement Planes (MP) positions, extracted from the compressor presented above. The first Measurement Plane became an inlet panel and the last MP equipped in probes became an outlet panel, what allowed to import the boundary conditions consistent with the test (e.g. inlet conditions are set as a circumferentially averaged radial profile, extracted from the corresponding MP at the specific operating conditions). Due to the specificity of compressor modelling i.e. Rotor-Stator configuration, interface connecting two frames of reference has been introduced. Rotating and Stationary frame of reference are connected with Mixing Planes. Mixing Plane approach performs a coupling between tangential averages on both sides of the interface between the adjacent rows each of which sees a tangentially constant flow field at the coupling interface. A change of the pitch, as well as periodicity, is supported by this kind of mixing procedure. Existing in a real machine bleedports and leakages has been omitted to reduce the model complexity and computing time.
Two different Two-Equation turbulence models were used to compute the results - Wilcox $1988 \mathrm{k}-\omega$ described in [15], and Menter's SST $2003 \mathrm{k}-\omega$ described in [16]. Aim was to find which model will show better fitting to the measurements using simplified model.

All performed computations received an initial flow conditions based on the two-dimensional results provided by Streamline Curvature Method [17] (adjusted to the Rig operating conditions in the same manner as a 3D model) which creates a complete aerodynamic solution of turbomachinery flow field, based on circumferential averaged values. Initialization provided with this approach reduces computational time and possible numerical problems.

\section{GEOMETRY AND MESHING}

Real geometry of the compressor includes fillets for all the rows. All Stators are equipped with Inner Air Seal (Labyrinth Sealing) - no cantilever Stators present. In addition bleed-port is mounted after Stator 4. For the model creation significant

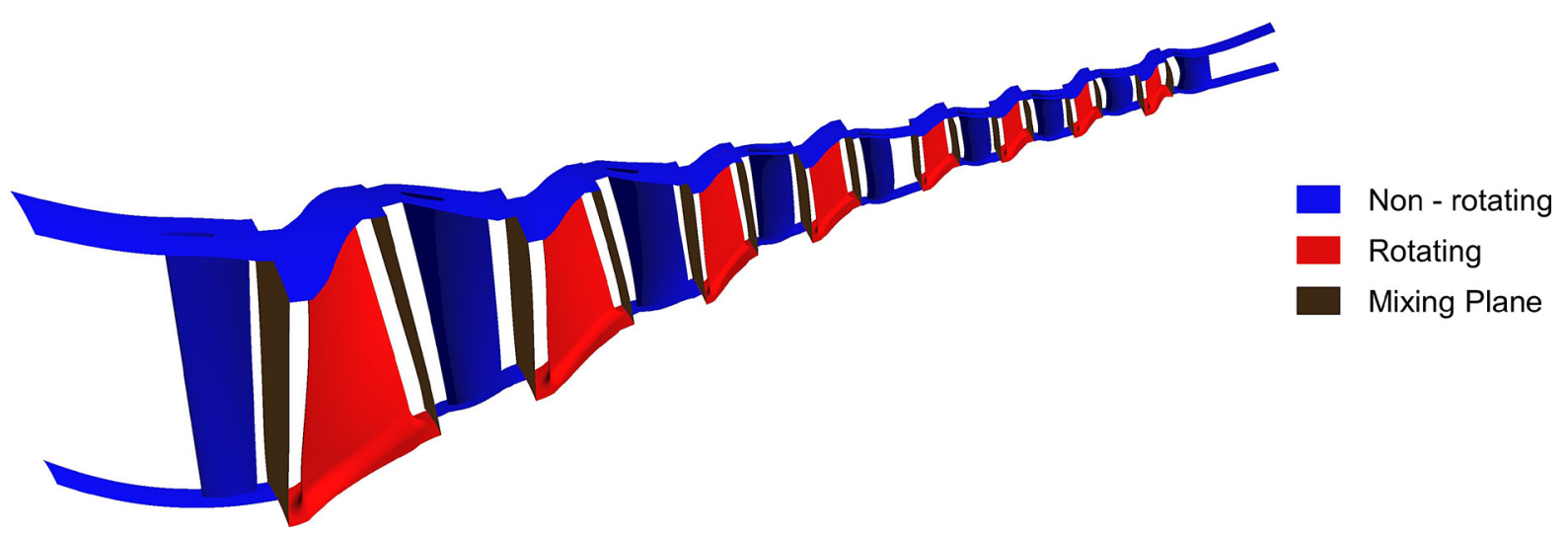

Fig. 2. Domain representation

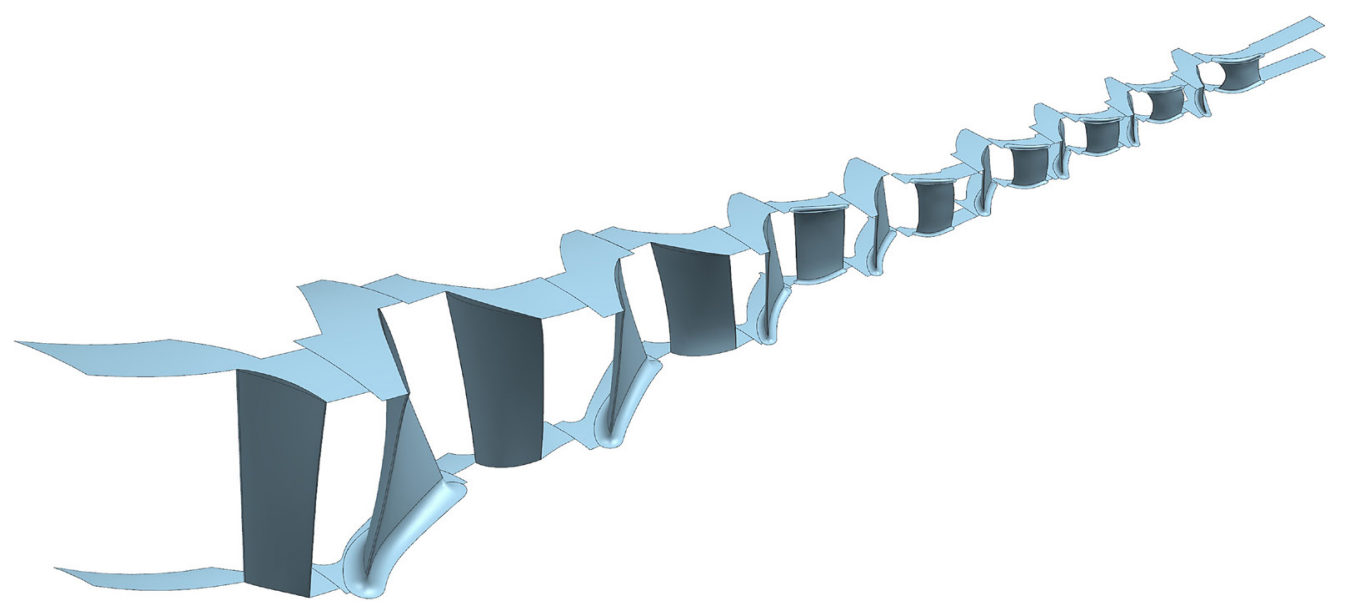

Fig. 3. Base geometry 


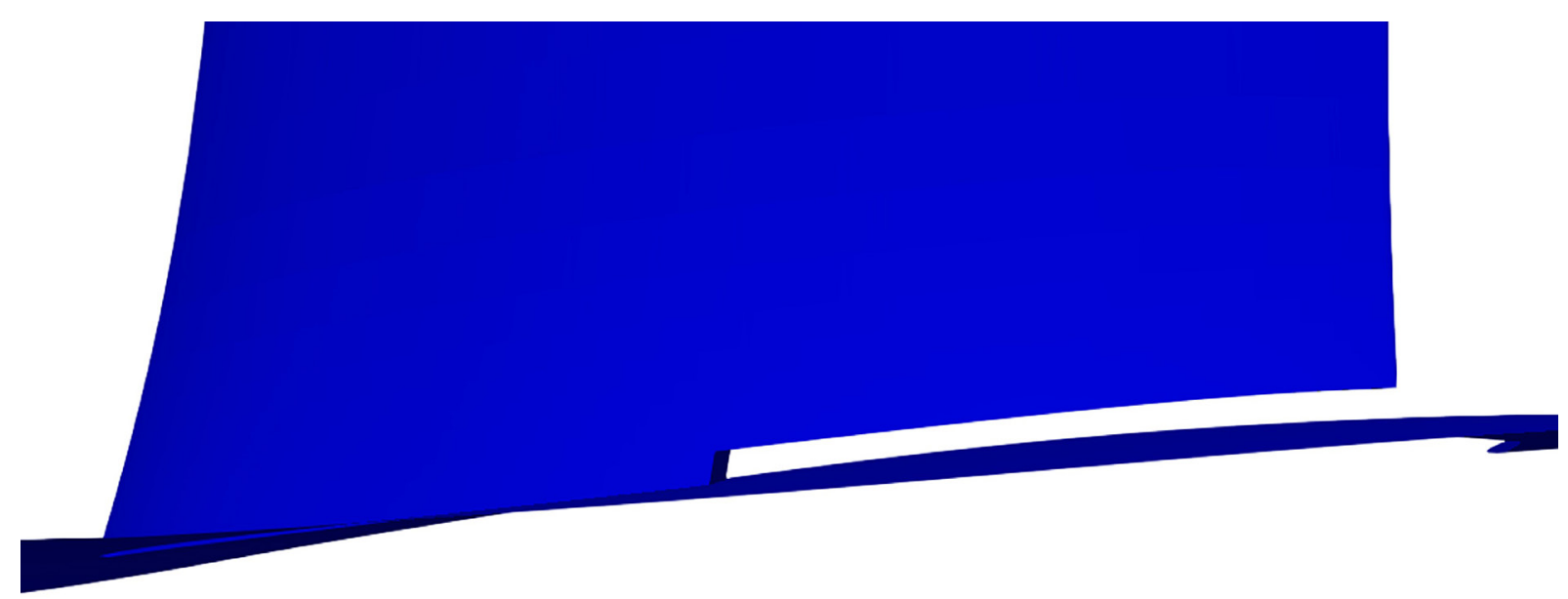

Fig. 4. Close look on a partial gap of S1

simplifications were introduced. Due to the fillet geometry influence on the secondary flow, all the rows are modelled with Rig corresponding radiuses. Implicitly well represented geometry will help with measurement data fitting especially in a range of stability limit. With known importance of tip clearances on SM prediction, gaps are modelled for all the Rotors. Additionally partial gaps of the VGV's are considered as well (Fig. 4.).

The Finite Volume discretization grid is created with use of commercial program AutoGrid developed by Numeca. It is a powerful meshing tool providing high-quality multi-block structured meshing for a variety of applications. AutoGrid streamlines the simulation workflow, provides a good mesh quality with optimal number of cells and allows the user to control a first cell size and gridlines aligned with the flow paths. For this specific work special settings were used. Three mesh sizes for three rotational speeds were introduced where target of wall was $\mathrm{y}+=1$. The reason of using constant $\mathrm{y}+$ along all the grid resolutions was to minimize discretization error at boundary layer. For passage meshing, o-block strategy was used with attention to Leading Edge (LE) and Trailing Edge (TE). To reduce the influence of the geometry discretization quality, similar node number was kept for all grid resolution at LE and TE regions (Fig. 5).

\section{MESH DEPENDENCY STUDIES}

Preliminary studies were aimed to define if used as reference mesh was independent and which turbulence model is better to predict the flow in the simplified model. All the studies were done for entire compressor. The approach of the compressor throttling was to increase static pressure at the outlet plane with the final increment of $0.1 \%$. That allowed to find the high quality last stable point based on the numerical stability. The first study was to find if current mesh is converged. For that reason Refined Fine Mesh was introduced. Statistics of the mesh density are presented below (Table 1).

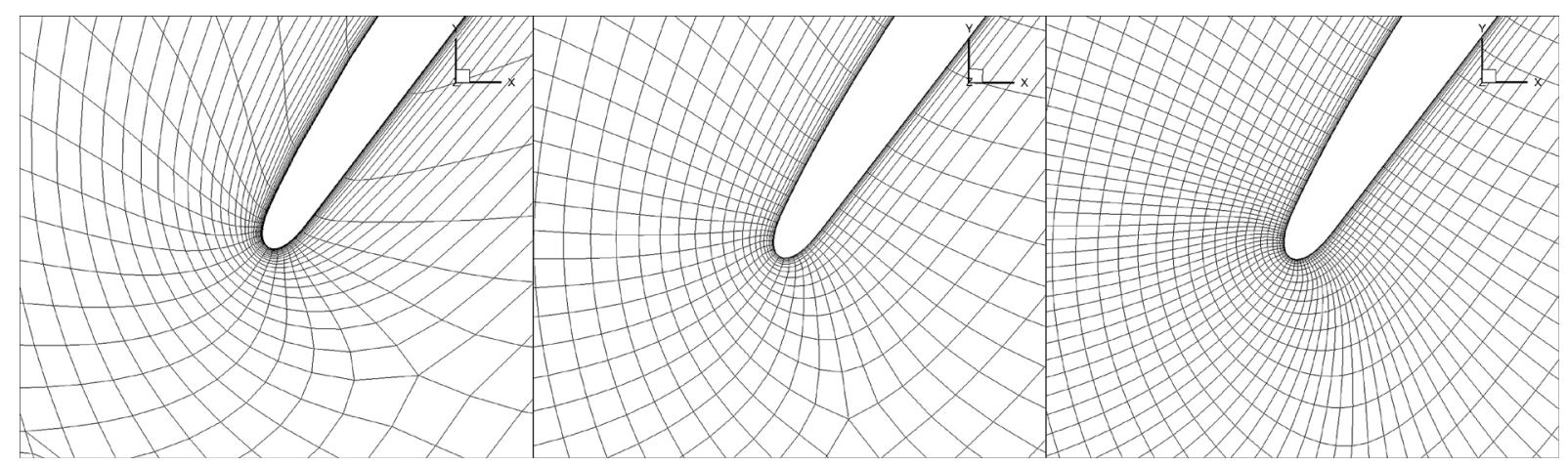

Fig. 5. LE in detail (from the left side: Coarse, Medium, Fine) 
Table 1. Mesh statistics

\begin{tabular}{|l|c|c|}
\hline \multicolumn{1}{|c|}{ Rotational speed } & Nodes (fine) [-] & Nodes (Extra fine) [-] \\
\hline Low speed & 11390860 & 16315334 \\
\hline Mid speed & 10719632 & 15277574 \\
\hline High speed & 10662928 & 15292726 \\
\hline
\end{tabular}

Table 2. Mesh convergence

\begin{tabular}{|l|c|c|}
\hline \multicolumn{1}{|c|}{ Rotational speed [\%] } & PR deviation (Fine-Extra Fine) [\%] & Mass Flow deviation (Fine to Extra Fine) [\%] \\
\hline Low speed & 0.00 & 0.032 \\
\hline Mid speed & 0.00 & 0.033 \\
\hline High speed & 0.00 & 0.013 \\
\hline
\end{tabular}

Basing on the close to Working Line (WL) operational point, Mesh Convergence analysis was performed. For the following study Wilcox k- $\omega$ turbulence model was used. The presented results revealed that no further grid refinement is needed.

As presented in the Table 2, Pressure Ratio (PR) is not sensitive for further grid refinement. Some deviations are observable at the Mass Flow nevertheless it was negligible and can be stated that Fine mesh is already independent, and can be used as a reference model.

Next step was to investigate how different turbulence models will behave through the speeds. Except variables fitting, shape of the Speed Lines was observed. For that, full up to the surge Speed Lines were performed. Turbulence model study was performed only for the converged mesh (Fine) with the assumption that both turbulence models will be grid converged at the same mesh resolution.
Both turbulence models were compared to the experiment data at the 3 different rotational speeds. Figures 6-8 shows that Menter's SST turbulence model is not well distributed through the speeds in compare to the measurement data and the Wilcox k- $\omega$ turbulence model. PR and Mass Flow shift is observed in all configurations. The biggest deviation is observed below High speed. Local fitment is not enough to state the best model definition. The Low speed SST result is physically not reasonable and forward bended Speed Line seems to be an artefact of the calculation. The decision was to use Wilcox k- $\omega$ turbulence model for the further considerations due to the more constant offset through the speeds. That will allow to find a factor for the bias.

The following studies were to find the best configuration (trade of between the mesh density and computing time) for the optimization

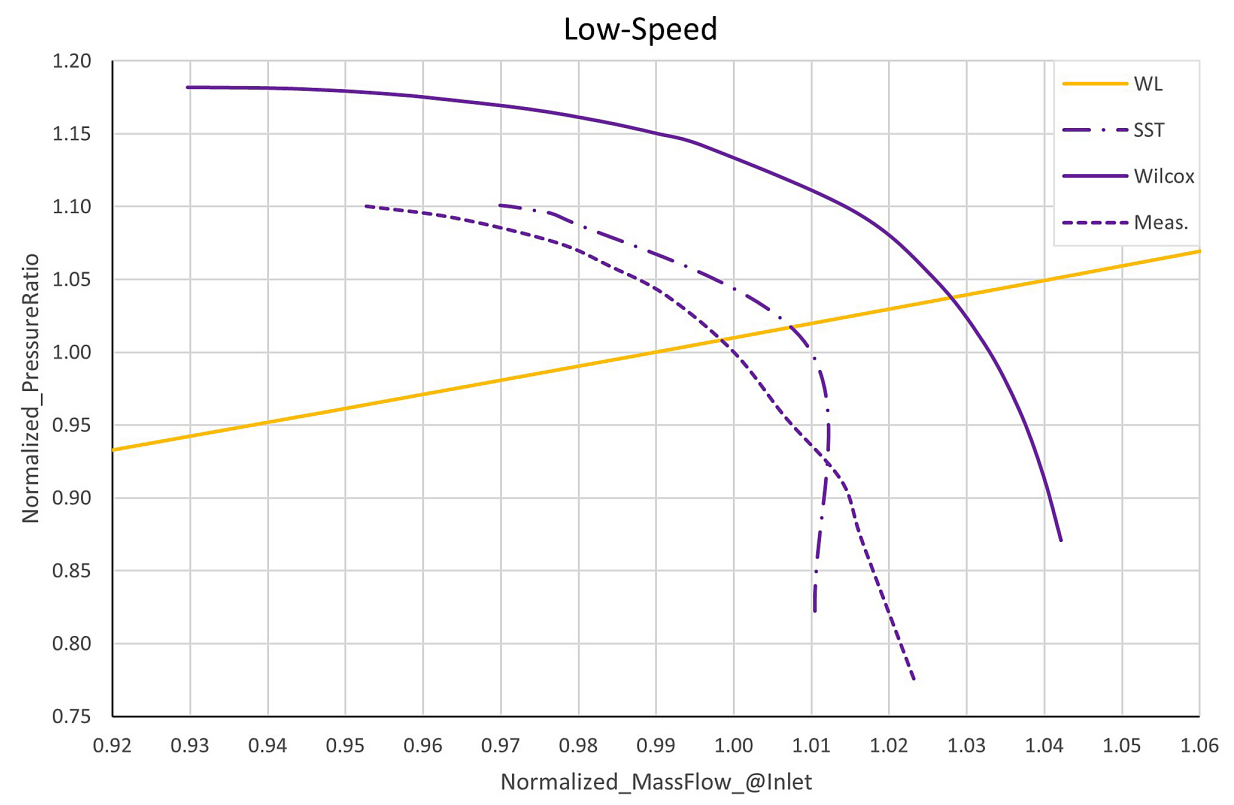

Fig. 6. Comparison of the turbulence models (Low speed) 


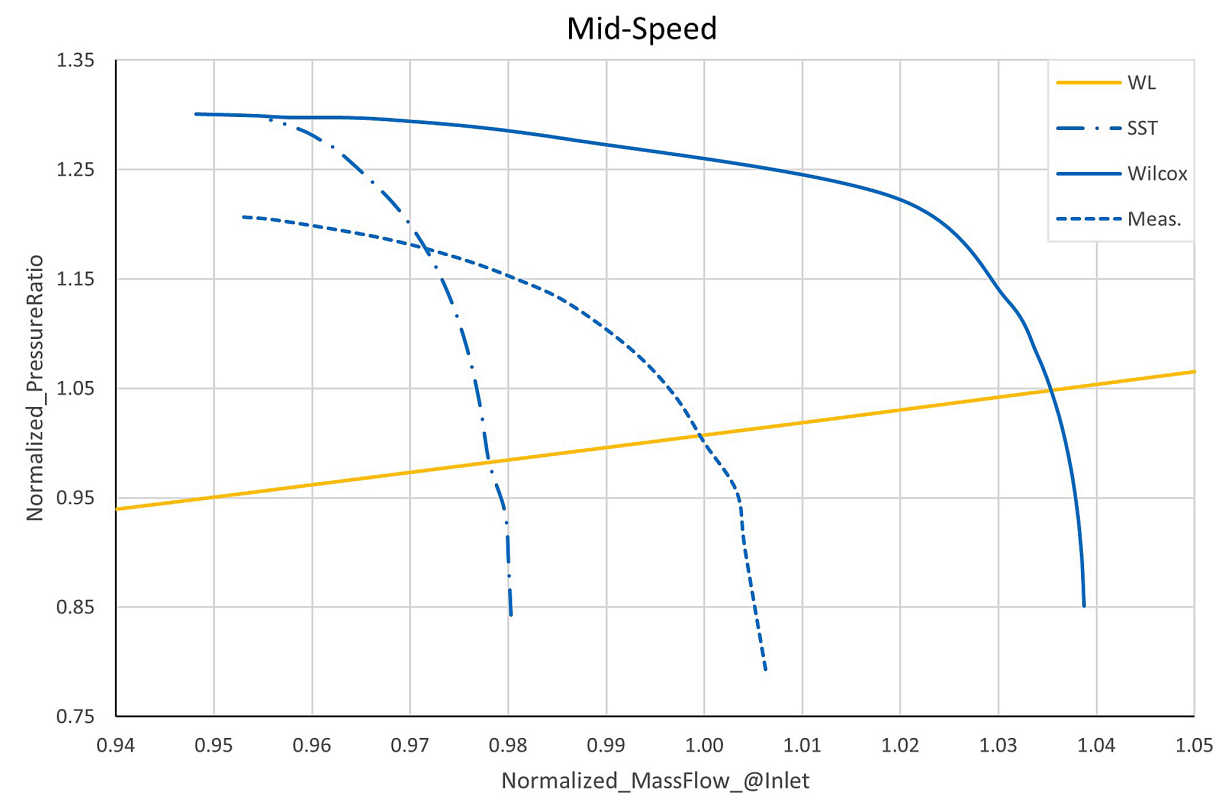

Fig. 7. Comparison of the turbulence models (Mid speed)

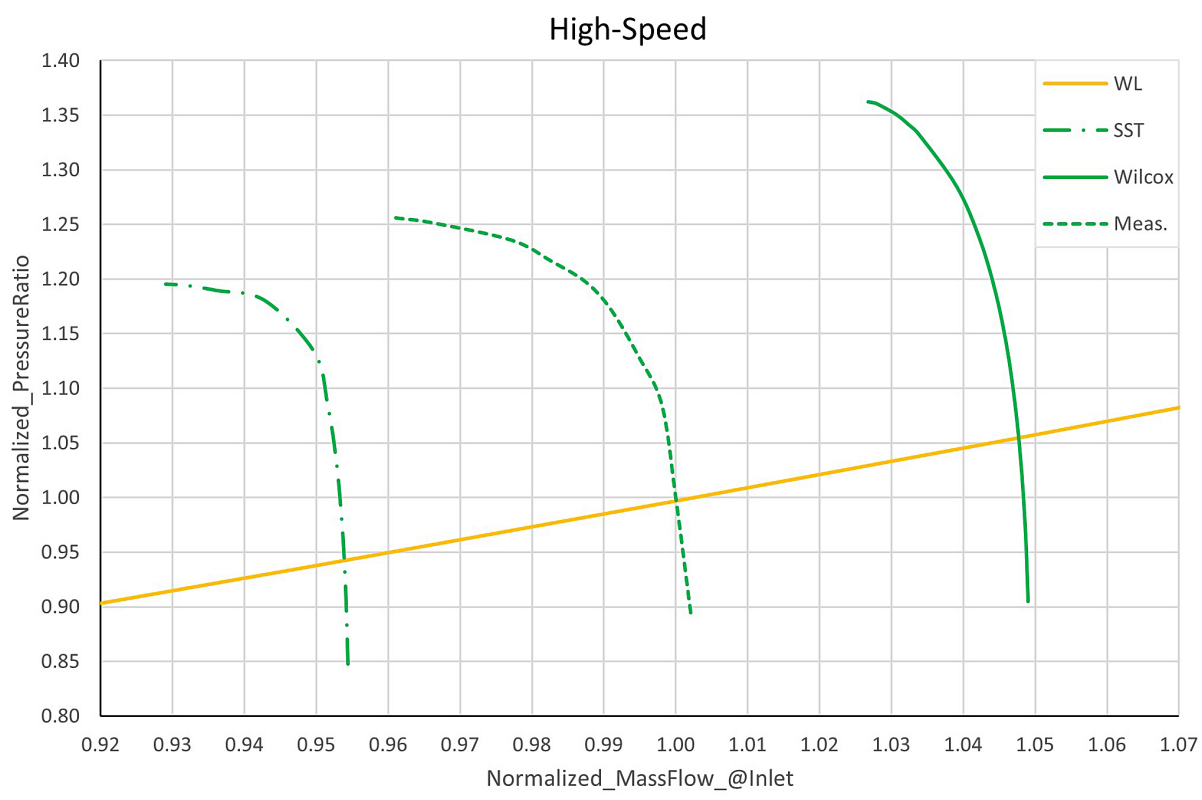

Fig. 8. Comparison of the turbulence models (High speed)

purposes. For that reason 3 different mesh resolutions were created. The coarse, medium and the fine one (node statistics shown in the Table 3). The target was to investigate how much the mesh can be coarsened to retain valuable data i.e. to achieve the mesh which will preserve the suitable characteristics where future bias approach can be applied. The configuration which demonstrate different Speed Line shape or significant PR and Mass Flow shift cannot be accepted. The following computations were performed.

Figures 9-11 shows set of Speed Lines for 3 rotational speeds. In compare to the Fine mesh all other configurations were showed shift in PR and Mass Flow. The sensitivity is not constant for every speed. The greatest inconsistency has been observed for the Mid speed (Fig. 10). As well as PR and Mass Flow shift, the shape of the Speed Line has changed for Coarse Grid. Averaged time reduction in case of Coarse configurations is visible (time reduced by about $60 \%$ to get converged results) but discrepancy of the information were not acceptable.

Due to the different mesh sensitivity along the rotational speed, Coarse mesh has been excluded 
Table 3. Numerical grid statistics

\begin{tabular}{|l|c|c|c|c|}
\hline \multicolumn{1}{|c|}{ Rotational speed } & Nodes (Coarse) [-] & Nodes (Medium) [-] & Nodes (Fine) [-] & Target $y+[-]$ \\
\hline Low speed & 3302589 & 5616126 & 11390860 & 1 \\
\hline Mid speed & 3282963 & 5376538 & 10719632 & 1 \\
\hline High speed & 3172653 & 5273786 & 10662928 & 1 \\
\hline
\end{tabular}

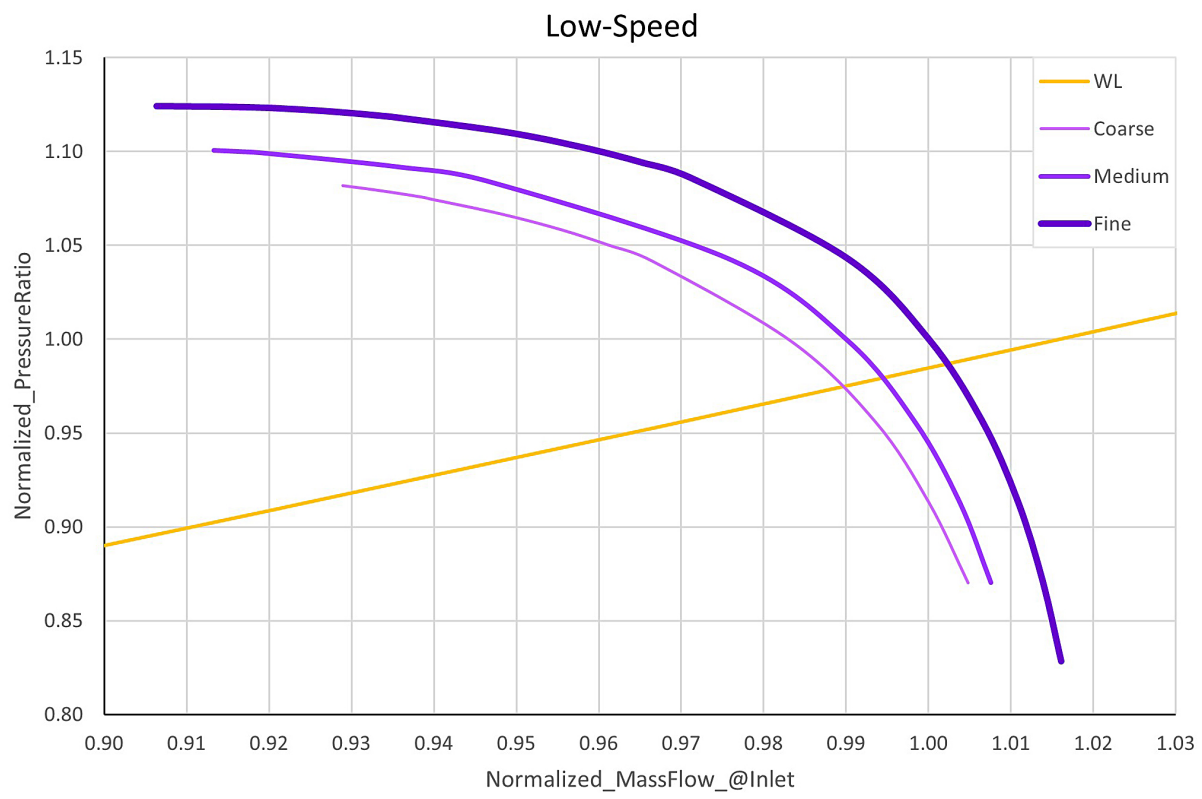

Fig. 9. Comparison of the mesh density (Low speed)

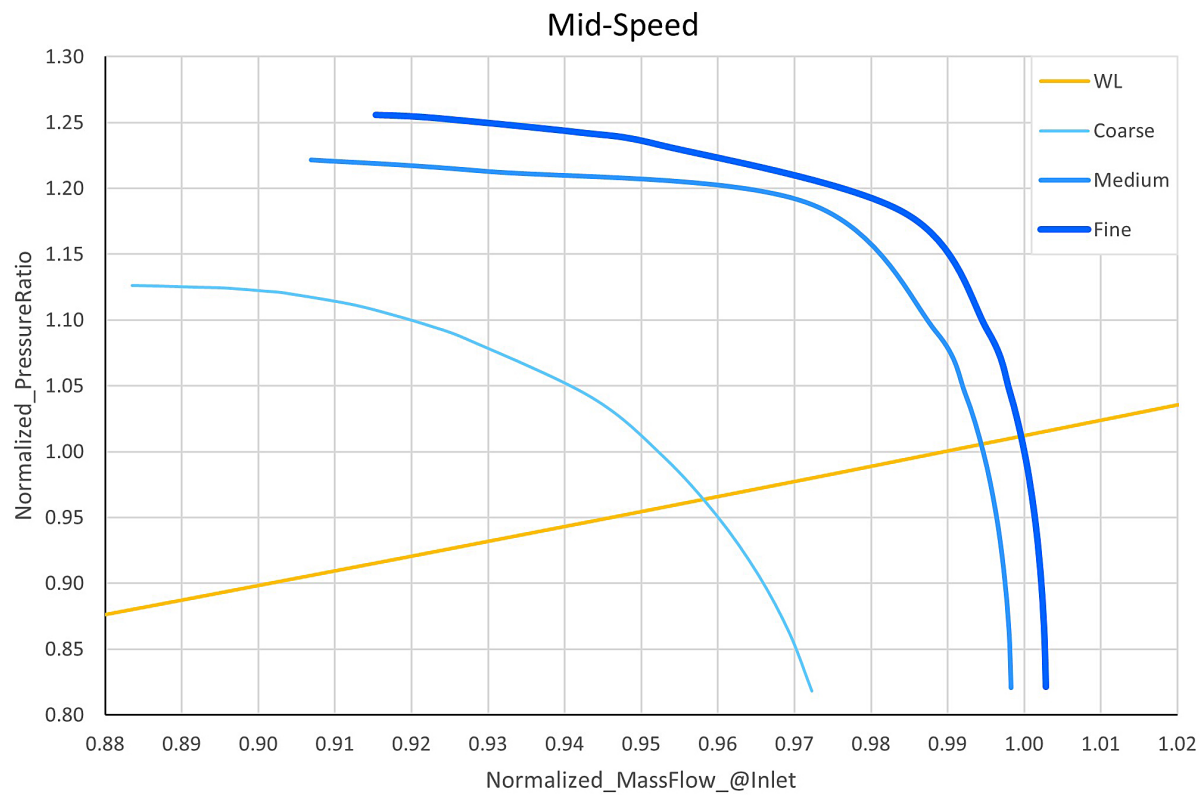

Fig. 10. Comparison of the mesh density (Mid speed)

from further investigation. The Medium mesh shows good representation of the characteristics shape. Major shift in the Mass Flow and PR has been observed but the goal of this work was to establish simplified configuration which can be used in the optimization process therefore some deviations can be accepted. To check how strongly mesh influences the internal flow and boundary layer, blockage level comparison has been performed. Blockage has been measured as volume of cells where axial Velocity were $\leq 0$. Results are presented in a Table 4. 


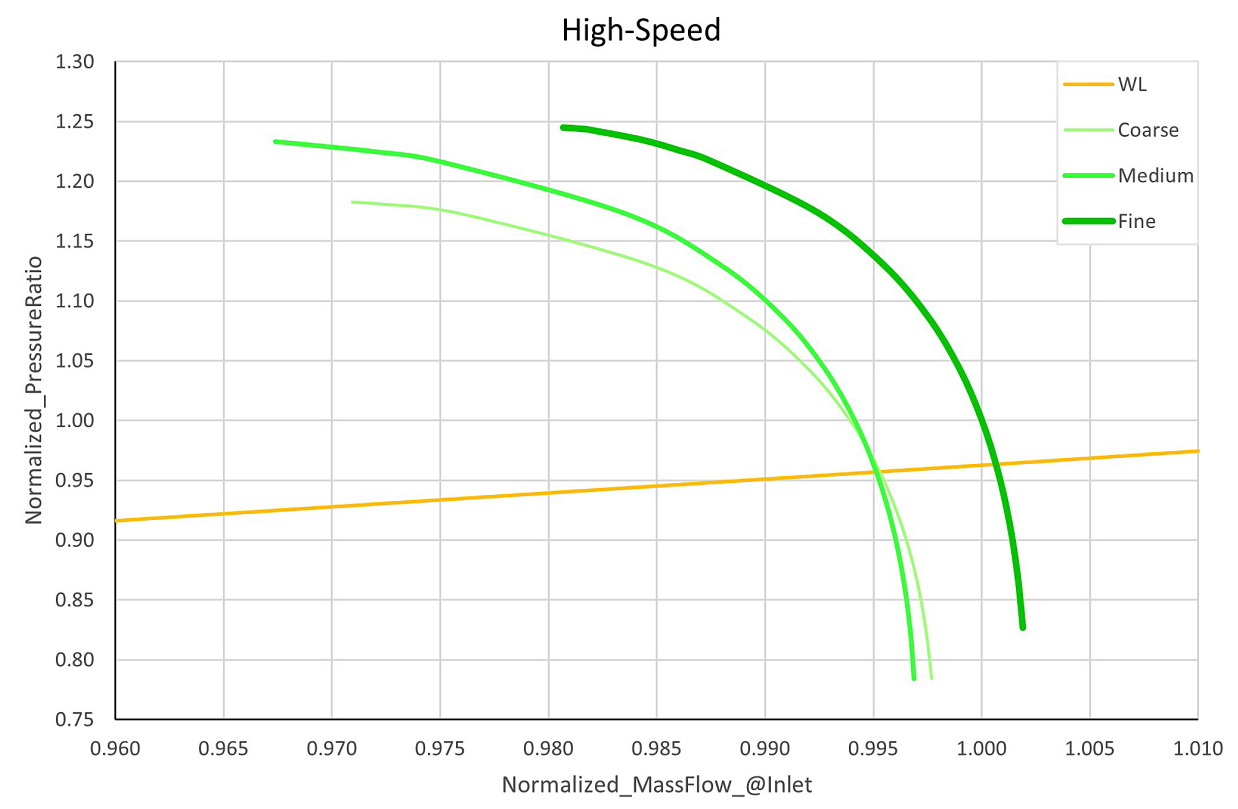

Fig. 11. Comparison of the mesh density (High speed)

Table 4. Blockage level comparison

\begin{tabular}{|l|c|c|c|}
\hline \multicolumn{4}{|c|}{ Blockage volume deviations in compare to Fine grid } \\
\hline \multicolumn{1}{|c|}{ Grid quality } & Low speed [\%] & Mid speed [\%] & High speed [\%] \\
\hline Coarse & 11 & 32 & 16 \\
\hline Medium & 4 & 6 & 11 \\
\hline
\end{tabular}

Again the Mid speed showed the biggest discrepancy for Coarse mesh. The strongest deviation of Medium grid was present at the High speed. This means that High speed is the most sensitive on the mesh variations. Time reduction of Medium grid has been reached - about 30\% to get converged results.
The last part was to check how meshes influenced the Surge Margin. There are many ways to define the surge margin properly. In this paper, one of the most common expressions was used and it is approach from Cumpsty [1]. He expresses the surge margin at the constant inlet Mass Flow where only the total Pressure Ratios are considered (1).

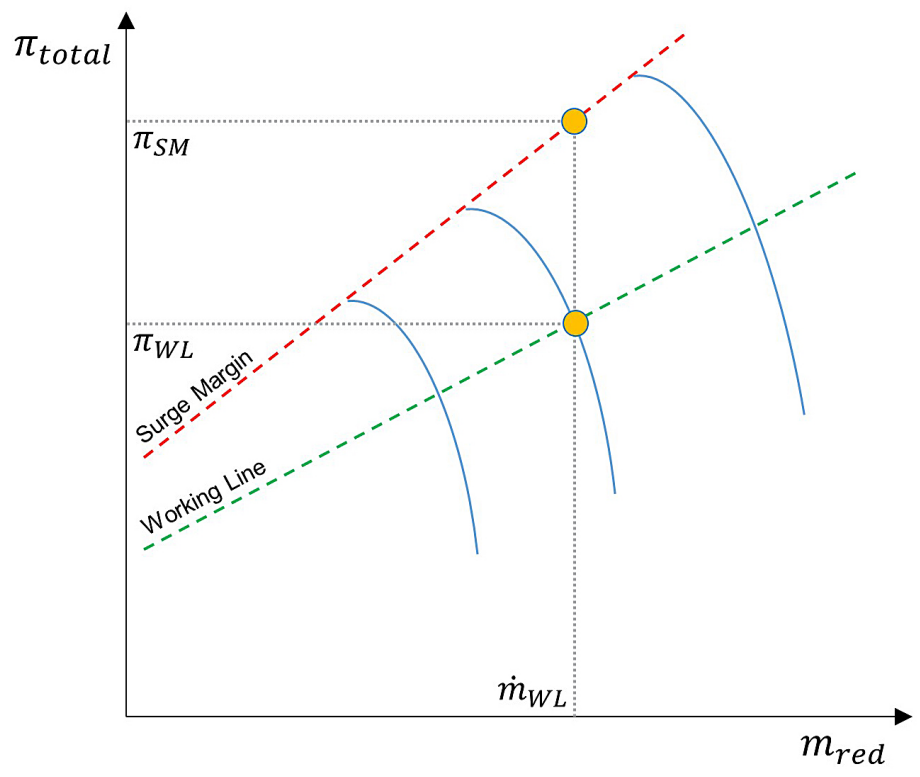

Fig. 12. Surge margin by pressure ratio definition 


$$
S M=\frac{\pi_{S M}-\pi_{W L}}{\pi_{W L}}
$$

Due to the PR and Mass Flow shift major displacement has been observed for the Surge Margin. Figure 13 shows how different resolution of meshes influences the Surge Margin. The lowest sensitivity for different densities of the grid was showed by High speed. The higher the rotational speed the better fitment is presented. Used SM definition has disadvantages, e.g. no Mass Flow shift is represented, but intention was to show the general inconsistency due to the model simplifications and mesh density provided.

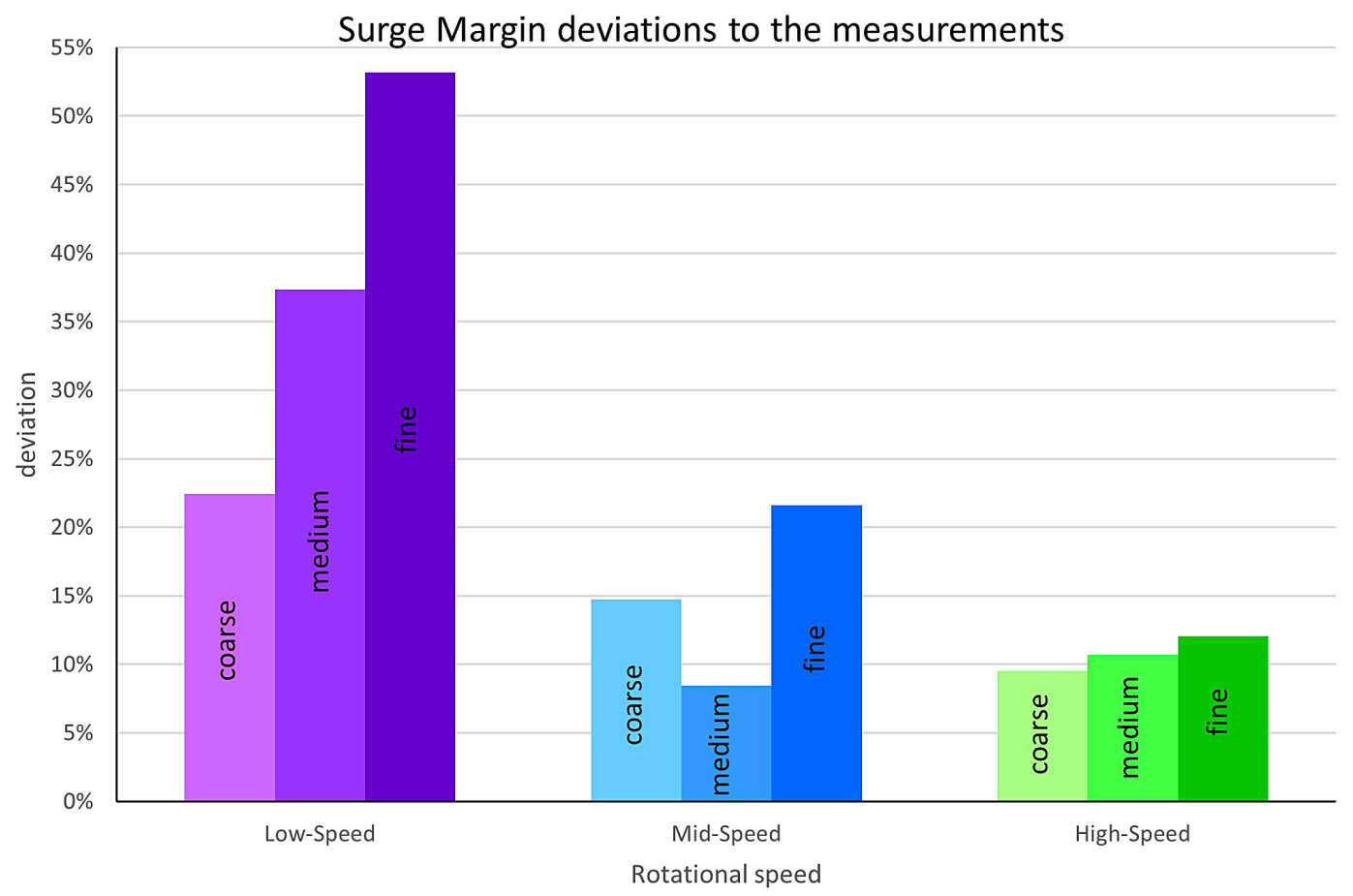

Fig. 13. Surge margin deviation in compare to the Measurements

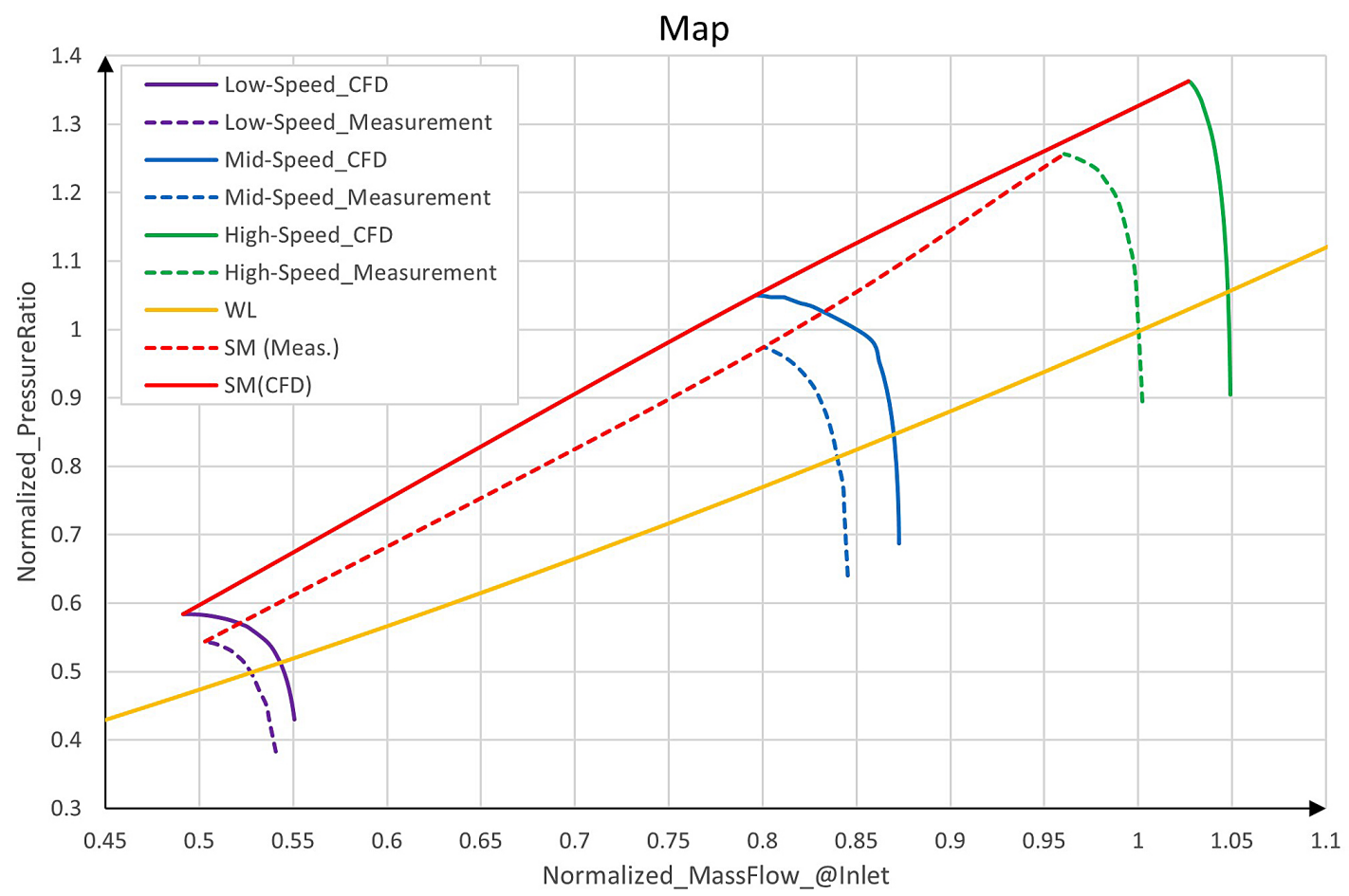

Fig. 14. Medium Grid and Measurements Surge Margin representation on a Compressor Map 
Because of intended use of the model (design and optimization) the Medium Grid with Wilcox $\mathrm{k}-\omega$ turbulence model was chosen for the future work. Due to the model simplifications even Fine grid showed the discrepancy to the Measurement data what was assumed. Basing on a fact that Medium Grid behaves in a similar way with reduced PR and Mass Flow, the result is to follow that approach. This will reduce the time by about $30 \%$ on each operational point, and trends out of the optimization shouldn't be affected by PR and Mass Flow discrepancy.

\section{CONCLUSIONS}

The results of conducted analyses were mesh dependency study, Measurements comparison, Speed Line plots, blockage volume comparison and general Compressor Map plot. All achieved results helped to find the best configuration for future optimization work.

As a results of conducted investigations the following conclusions were defined:

1. Neglecting the geometrical features like bleedports, leakages and labyrinth sealing significantly influences the Measurements data representation.

2. Wilcox $k-\omega$ showed better representation of the Measurements data than Menter's SST. For all rotational speeds shift consistency has been shown in opposite to Menter's SST.

3. Mesh density influence is not consistent at all rotational speeds.

4. Mesh coarsening have to be conducted separately with respect to all considered speeds.

5. For further optimization purposes, the Medium Grid with Wilcox k- $\omega$ turbulence model has been chosen as a good trade-off configuration.

\section{Acknowledgements}

The research presented in this paper was performed in collaboration with MTU Aero Engines AG and supported by Department of Aerospace Engineering, Faculty of Mechanical Engineering and Aeronautics, Rzeszow University of Technology in favour of doctoral dissertation carried out under polish industrial doctorate program.

\section{Nomenclature}

\begin{tabular}{|l|l|}
\hline CFD & Computational Fluid Dynamics \\
\hline DLR & $\begin{array}{l}\text { Deutsches Zentrum für } \\
\text { Luft- und Raumfahrt } \\
\text { (German Aerospace Center) }\end{array}$ \\
\hline DP & Design Point \\
\hline EARSM & $\begin{array}{l}\text { Explicit Algebraic Reynolds } \\
\text { Stress Model }\end{array}$ \\
\hline HPC & High Pressure Compressor \\
\hline IFSD & In-Flight Shut Down \\
\hline IGV & Inlet Guide Vane \\
\hline LE & Leading Edge \\
\hline MP & Measurement Plane \\
\hline N & Rotational speed \\
\hline PR & Pressure Ratio \\
\hline RANS & Reynolds-Averaged Navier-Stokes \\
\hline R1 & Rotor 1 \\
\hline SM & Surge Margin \\
\hline SST & Shear Stress Transport \\
\hline S1 & Stator 1 \\
\hline TE & Trailing Edge \\
\hline TRACE & Namics Computational Environment \\
\hline VGV & Working Line \\
\hline WL & Non-dimensional wall distance \\
\hline 3D & Thrensional \\
\hline
\end{tabular}

\section{REFERENCES}

1. Cumpsty N.A. Compressor aerodynamics. Longman Scientific \& Technical; 1989.

2. Galloway L., Spence S., Kim S.I., Rusch D., Vogel K., Hunziker R. An Investigation of the Stability Enhancement of a Centrifugal Compressor Stage Using a Porous Throat Diffuser. Journal of Turbomachinery. 2018; 140.

3. Yang M., Zheng X., Zhang Y., Bamba T., Tamaki H., Huenteler J., Li Z. Stability Improvement of High-Pressure-Ratio Turbocharger Centrifugal by Asymmetric Flow Control- Part I: Non- Axisymmetrical Flow in Centrifugal Compressor. Journal of Turbomachinery. 2013; 135.

4. Möller D., Schiffer H.P. On the Mechanism of Spike Stall Inception and near Stall non-synchronous vibration in an Axial Compressor. In: ASME Turbo Expo 2020: Turbomachinery Technical Conference in Exposition. London, United Kingdom 2020.

5. Day I.J. Stall, Surge and 75 Years of Research. Journal of Turbomachinery. January 2016; 138.

6. Righi M., Pachidis V., Könöszy L., Pawsey L. Three-dimensional through-flow modeling of axial 
flow compressor rotating stall and surge. Aerospace Science and Technology. 2018; 78: 271-279.

7. Erler E., Vo H.D., Yu H. Desensitization of Axial Compressor Performance and Stability to Tip Clearance Size. Journal of Turbomachinery. 2016; 138.

8. Goodhand M.N., Miller R.J. The Impact of Real Geometrics on Three-Dimensional Separations in Compressors. Journal of Turbomachinery. March 2012; 134(2).

9. Salunke N.P. Design Opitimization of an Axial Flow Compressor for Industrial Gas Turbine. International Journal of Research in Engineering and Technology. May 2014; 3(5): 458-464.

10. Zheng R., Xiang J.H., Sun J. Blade Geometry Optimization for Axial Flow Compressor. In: ASME Turbo Expo 2010: Power for Land, Sea and Air. Glasgow, United Kingdom 2010; 633-644.

11. Ratz J., Leichtuß S., Beck M., Schiffer H.P., Fröchling F. Surge Margin Optimization of Centrifugal Compressor Using a New Objective Function Based on Local Flow Parameters. International Journal of Turbomachinery Propulsion and Power. 2020; 4: 42.

12. Jin-Hyuk K., Kwang-Jin C., Kwang-Yong K. Aerodynamics analysis and optimization of a transonic axial compressor with casing grooves to improve operating stability. Aerospace Science and Technology. 2013; 29; 81-91.

13. Belami T., Galpin P., Braune A., Cornelius C. CFD Analysis of a 15 Stage Axial Compressor: Part IMethods. In: ASME Turbo Expo 2005: Power for Land, Sea and Air. Reno, Nevada, United States of America 2005, 1001-1008.

14. Cornelius C., Biesinger T., Galpin P., Braune A. Experimental of Computational Analysis of a Multistage Axial Compressor Including Stall Prediction by Steady and Transient CFD Methods. Journal of Turbomachinery. 2013; 136(6).

15. Wilcox D.C. Turbulence Modeling for CFD. DCW Industries, Inc., 1993.

16. Menter F.R., Kuntz M., Langry R. Ten Years of Industrial Experience with the SSt Turbulence Model. Turbulence, Heat and Mass Transfer 4, ed: Hanjalic K., Nagano Y., Tummers M. Neell House, Inc., 2003; 625-632.

17. Novak R.A. Streamline Curvature Computing Procedures for Fluid-Flow Problems. ASME. J. Eng. Power. 1967; 89(4): 478-490. 